\title{
A semi-analytical model for the temperature distribution of thermo-inductive heating
}

by B. Oswald-Tranta*, G. Wally*, J. Oswald**

*Institute for Automation, University of Leoben, Austria

** Institute for Physics, University of Leoben, Austria

\begin{abstract}
In the case of thermo-inductive testing the material is heated by induced eddy currents and the emission from the material surface is detected by an infrared camera. Anomalies in the surface temperature correspond to in-homogeneities in the material. Experiments show that for magnetic steel the edges of surface cracks become visible through higher temperatures. In contrast, in stainless steel one observes lower temperatures around a surface crack. A semi-analytical model has been developed to describe this phenomenon. After calculating the distribution of the induced eddy current around the crack, the generated Joule-heat along the current streamlines is determined. The temperature distribution is calculated according to the heat conductivity. Additionally, finite element simulations have been carried out, in order to model the temperature distribution caused by the eddy currents. The comparison of the calculated and measured results shows very good agreement.
\end{abstract}

\section{Introduction}

Thermo-inductive probing $[1,2,3,4,5,6,7]$ is a thermo-graphic non-destructive testing method to detect shallow surface cracks in metallic materials. The technique uses induced eddy currents to heat the material being tested. The heat dissipation due to the eddy current and the resistance of the material causes local heating. The infrared emission from the material surface is detected by a suitable imaging system. Anomalies in the surface heating correspond to defects at the surface. The technique can be used for the inspection and detection of macroscopic cracks in materials where eddy currents can be induced, whereby metals are particularly well suited.

In experiments it has been observed, that in the case of magnetic materials the surface cracks exhibit an additional temperature increase around the cracks. It has been shown [7] that the deeper the surface crack is, the higher is the temperature increase at the edges of the crack. An analytic expression has been derived $[7,8]$, which allows the crack-depth to be determined from the measured temperature increase.

In the case of non-magnetic materials experiments show that a lower temperature occurs at the edges of the cracks and high temperatures at the tip of the crack $[5,6]$. The goal of this paper is to model the eddy current distribution inside the material and calculate the temperature distribution around long surface cracks for different parameters. The boundary conditions for getting either lower or higher temperatures at the edges of the surface cracks are investigated. 


\section{Experimental results}

Thermo-inductive measurements have been carried out on different workpieces. The experimental setup is shown in Figure 1, whereas the tested magnetic steel is inserted into an induction coil. There are two surface cracks with a depth of about 1-3mm along the piece. The infrared camera is looking at the heated steel probe through the free space of the coil windings.

The camera is triggered externally to record a thermal image after a well defined very short heating period. Figure 2 shows the infrared image after $0.1 \mathrm{sec}$ heating. The surface cracks and the corners of the workpiece become additionally warmer than the steel sample itself.

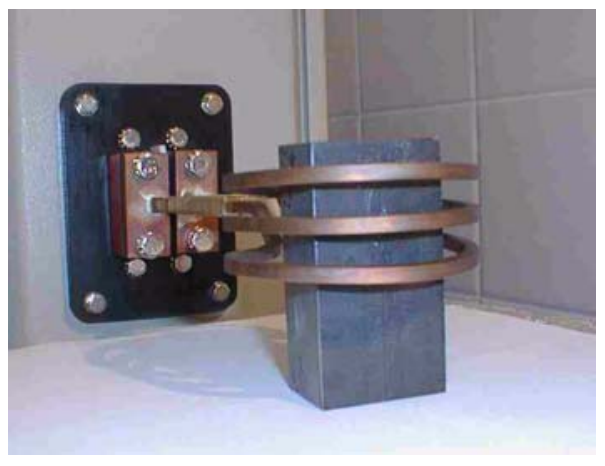

Figure 1: Inductive heating of a steel workpiece inserted into the induction coil.

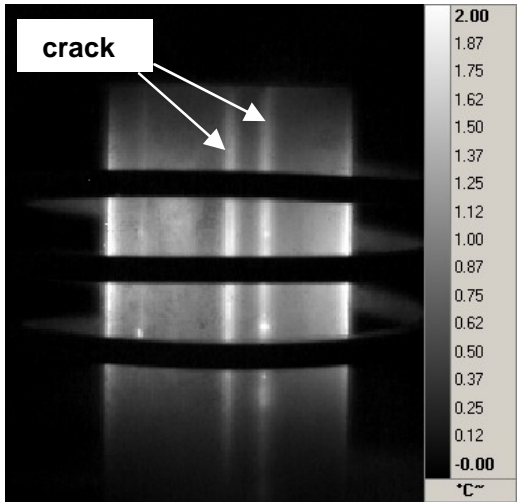

Figure 2: Infrared image of the heated steel shown in Figure 1.

Figure 3 shows the infrared image of an inductive heated stainless steel wire. The shallow surface crack along the wire can be seen very well by the lower temperature as compared to the surrounding surface. These experiments demonstrate that in magnetic materials the crack is visible by higher temperatures and in non-magnetic materials by lower temperatures.

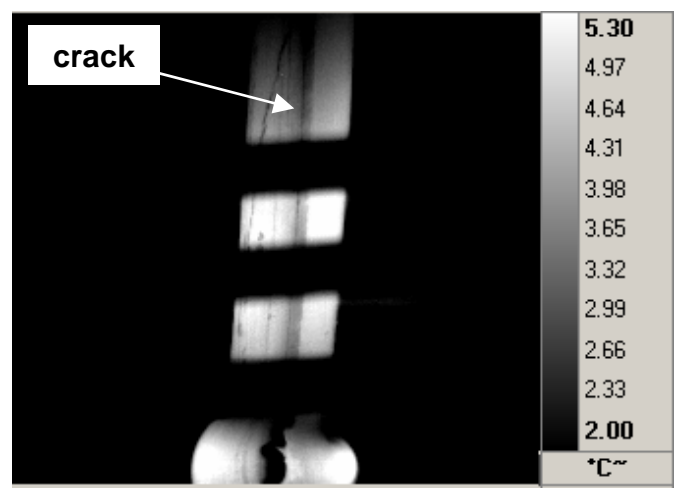

Figure 3: Infrared image of the heated stainless steel wire. 
Further experiments have been carried out on sheet plates of steel with low magnetic permeability. The plate has been placed in the middle of an induction coil. Figure 4 shows the infrared image after a short heating period. The sample is a long rectangle where a notch with a depth of $2 \mathrm{~mm}$ has been cut into the sheet. The tip of the notch is visible by a small circular high temperature region, but the corners of this crack are colder than the sides of the rectangle. In the case of thermo-inductive testing of long pieces as shown in Figure 3, we don't see the hot tip of the surface crack, which is hidden, but we see only the temperature of the surface that means the lower temperature at the edge of the crack.

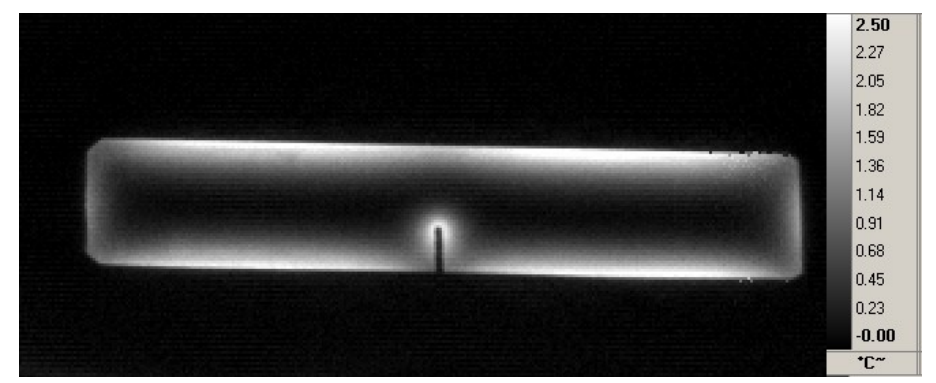

Figure 4: Infrared image of the heated steel sheet with an artificial crack

\section{Modelling of the eddy current distribution}

For the inductive heating mid- and high frequency $(20-300 \mathrm{kHz})$ is used to generate the eddy current. Because of the well-known skin effect the eddy current decays exponentially below the surface. The coefficient of this exponential function, the so-called penetration depth $\delta$, can be calculated by the following formula:

$$
\delta=\sqrt{\frac{1}{\pi \sigma \mu \mu_{0} \nu}}
$$

where $\sigma$ is the electrical conductivity, $\mu$ is the relative permeability, $\mu_{0}$ is the permeability of vacuum and $v$ is the excitation frequency. Magnetic steel with high relative permeability has a very small penetration depth of about $0.03 \mathrm{~mm}$ at $200 \mathrm{kHz}$. In this case the penetration depth of the eddy current can be mainly neglected compared to the diffusion length of the heat flow. Therefore, the heat generation can be simply modelled by assuming the heat to be generated directly at the surface of the sample [7]. For non-magnetic materials the penetration depth of the eddy current is much larger (about $0.8 \mathrm{~mm}$ at $200 \mathrm{kHz}$ ), therefore the eddy current distribution has to be taken into account for modelling of the heat generation.

The exponential function is strictly valid only for a semi-infinite long plain metal surface [9]. For other geometries the distribution of the eddy current has to be calculated in a more complicated way. A good estimation for the eddy current distribution around a surface crack can be achieved with the aid of an appropriate conform transformation. It is transforming a Cartesian grid line system to the geometry of the surface crack, while keeping the angle of $90^{\circ}$ between the grid lines. 
By using the complex function [9]:

$$
z=\sqrt{w^{2}-d^{2}}
$$

where $w$ the original and $z$ transformed complex numbers are and $d$ is the depth of the crack. The horizontal lines (representing complex numbers with constant imaginary part) are transformed into the lines shown in Figure 5. One can see, that the transformed lines don't penetrate into the corners, but densify around the tip of the crack, just like what one has to expect from the streamlines of the eddy currents which have to pass the crack.

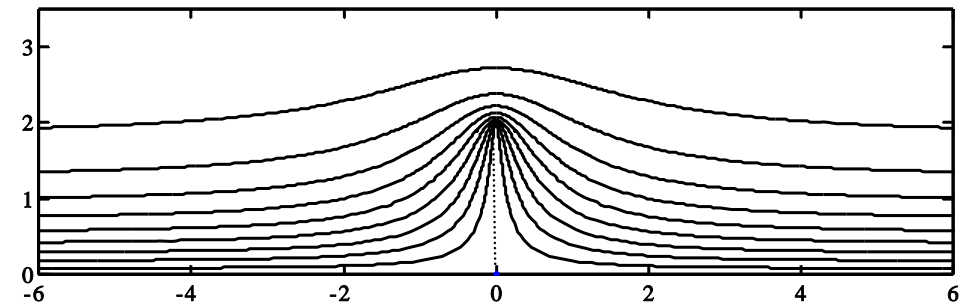

Figure 5: Calculated current streamlines around a surface crack with a depth of $2 \mathrm{~mm}$ at the position of $(0,0)$.

In a second step the generated heat along the streamlines is determined. The calculation is carried out numerically and dividing the whole sample into elements along the streamlines. In the corner of the crack there is a larger distance between the streamlines, indicating a lower current density and thus the locally generated Joule-heating becomes less in this region. In contrast, at the tip of the crack the streamlines densify, which indicates a high current density and a high local heating. In the last step of the calculation the propagation of the heat is determined according to the heat conductivity of the material. Heat exchange with the environment and across the crack can be neglected because of the short time to be considered and the high heat conductivity of metals. The heat which is generated in the corner of the crack cannot flow across the crack, therefore after a longer heating period it accumulates in the corner. Using this model the temperature distribution around a crack is calculated, as shown in Figure 6. The comparison with the experimental results (depicted in Figure 4) shows a good agreement.

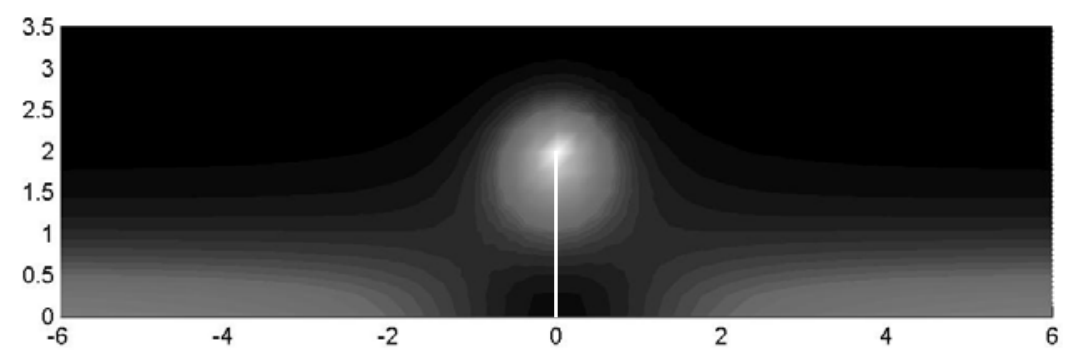

Figure 6: Calculated temperature around a surface crack with a depth of $2 \mathrm{~mm}$ at the position of $(0,0)$. 


\section{Numerical simulations for a surface crack}

Numerical simulations have been carried by the multi-physics finite element simulator ANSYS [10]. With this simulator it is possible to combine the modelling of electrical and thermal phenomena. In the first step the induced eddy current and the local Joule heating is calculated. In the second step the distribution of the heat is determined by the thermal module.

Figures 7 and 8 show isolines of the magnetic field around a surface crack with a depth of $1 \mathrm{~mm}$. Using the Maxwell-equations one can show, that in the case of a very long work piece these lines correspond to the streamlines of the eddy current. Figure 7 shows the streamlines for a penetration depth of $1 \mathrm{~mm}$ and Figure 8 for $0.1 \mathrm{~mm}$.

One can see, that for a penetration depth of the eddy current which is comparable with the depth of the crack (see Figure 7), the current is pushed out from the edges of the crack into the material, causing a lower current density at the edges of the crack. The opposite appears near the tip of the crack, resulting in a higher current density. Therefore, after only a very short heating period the tip of the crack is warmer, but the edge of the crack is colder than the surface of material, see Figure 9. After a longer heating period the initial temperature distribution is smeared out by the heat flow and gets more or less homogenously distributed along the side of the sample, see Figure 10.

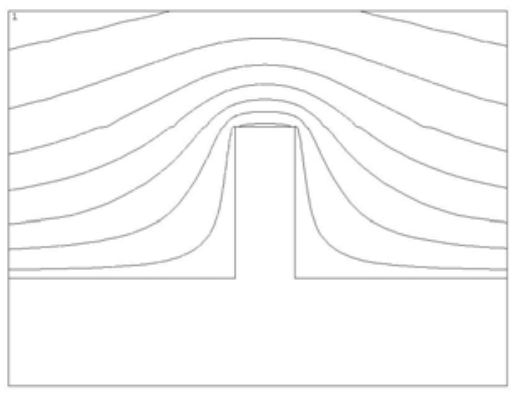

Figure 7: Calculated isolines of the magnetic field around a surface crack with a depth of $1 \mathrm{~mm}$ (penetration depth of the eddy current is $1 \mathrm{~mm}$ ), which at the same serve as the streamlines for the eddy currents.

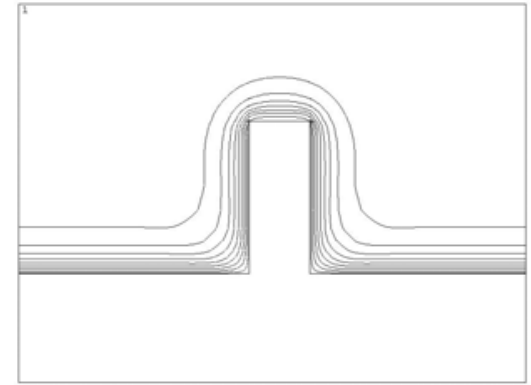

Figure 8: Same as Figure 7, but for a penetration depth of the eddy current of $0.1 \mathrm{~mm}$

If the penetration depth of the eddy current is significantly smaller than the depth of the surface crack, the current is following more or less along the sides of the crack, see in Figure 8. Therefore, the edge of the crack becomes also warmer already after a very short heating duration, see Figure 11. 


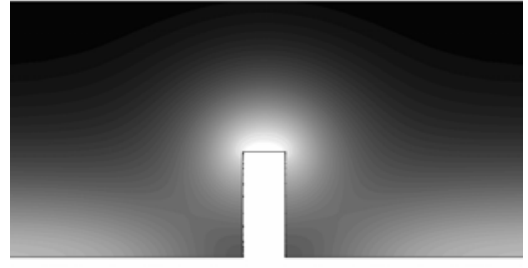

Figure 9: Calculated temperature distribution around a surface crack with a depth of $1 \mathrm{~mm}$ after $0.01 \mathrm{sec}$ inductive heating. The penetration depth of the eddy current is $1 \mathrm{~mm}$.

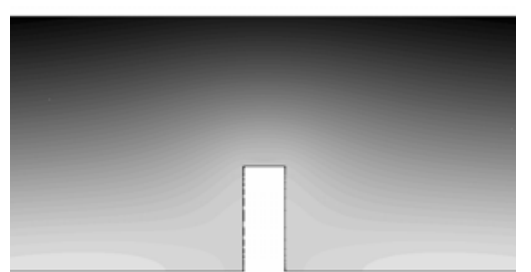

Figure 10: Calculated temperature distribution for the same case as shown in Figure 9, but after a heating period of $0.4 \mathrm{sec}$.

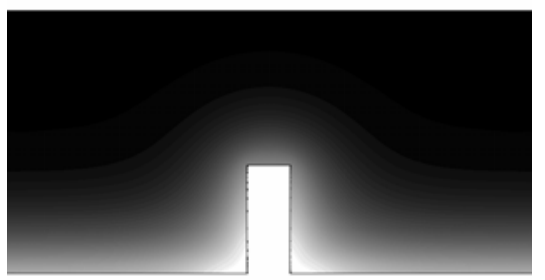

Figure 11: Calculated temperature distribution around a surface crack with a depth of $1 \mathrm{~mm}$ after $0.01 \mathrm{sec}$ inductive heating. The penetration depth of the eddy current is $0.1 \mathrm{~mm}$.

Figure 12 shows the ratio between the temperature increase at the edge of the crack $\left(\Delta T_{c}\right)$ and the temperature increase at the side of the sample $\left(\Delta T_{s}\right)$ versus the heating period. If the penetration depth is $1 \mathrm{~mm}$, then the ratio is increasing with the heating duration, but it remains less than 1 . This means that at the beginning the corner of the crack is colder than the side, but after some time the temperature becomes more or less uniformly distributed at the surface. These results are in very good agreement with the experimental results, shown in Figure 3.

In the case of a small penetration depth of $0.1 \mathrm{~mm}$, the corner of the crack is even warmer than the side already after a short heating period, therefore the ratio is larger than 1, like shown in Figure 12 by the dashed line. With increasing heating time the ratio becomes smaller, which means, that the additional temperature increase around the crack becomes less. These simulation results are also in very good agreement with the experimental results, shown in Figure 2. 
http://dx.doi.org/10.21611/qirt.2006.034

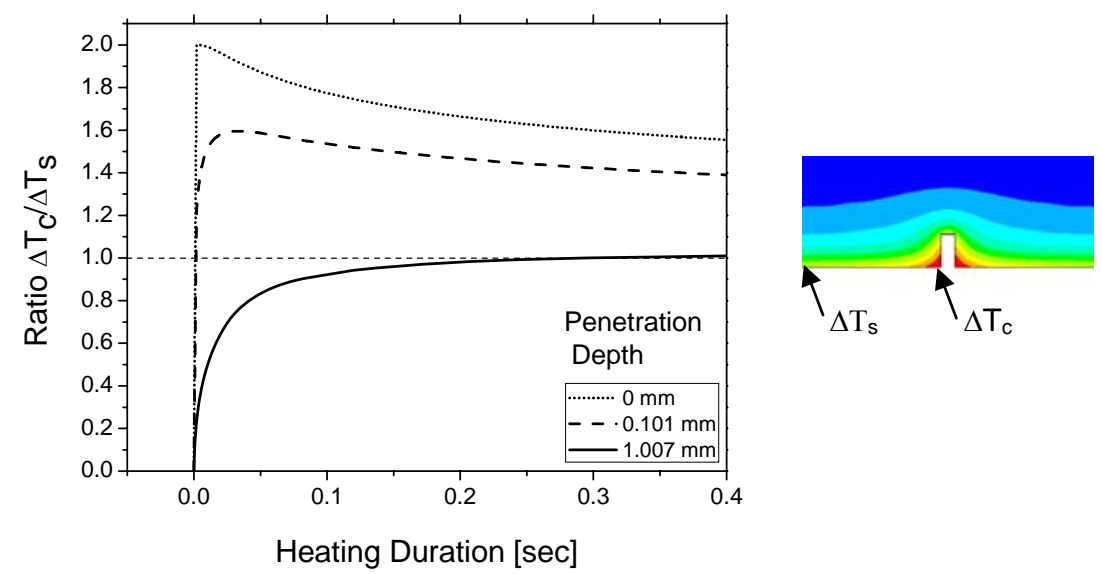

Figure 12: Ratio between the temperature increase in the corner of the crack and at the side of the sample as a function of the heating period for different penetration depth values. The insert shows the definition of the temperatures.

If the penetration depth is negligible small, then the calculation can be done with the simplified model, that the heat is applied directly at the surface of the sample. For this case the equations for describing the temperature distribution around a surface crack have been derived in [7] and [8]. Using these equations the dotted curve in Figure 12 has been calculated for the theoretical limit of a zero penetration depth. This curve shows, that immediately after switching on the inductive heating, the temperature ratio is 2 and with increasing time it decreases. Figure 13 shows the temperature ratio for different crack depth values, calculated by the analytical model $[7,8]$ which means introducing the heat directly to the surface of the sample. One can see that the smaller the crack is, the earlier the additional heating around the crack diminishes, because a shallower crack is passed more quickly by the heat flow.

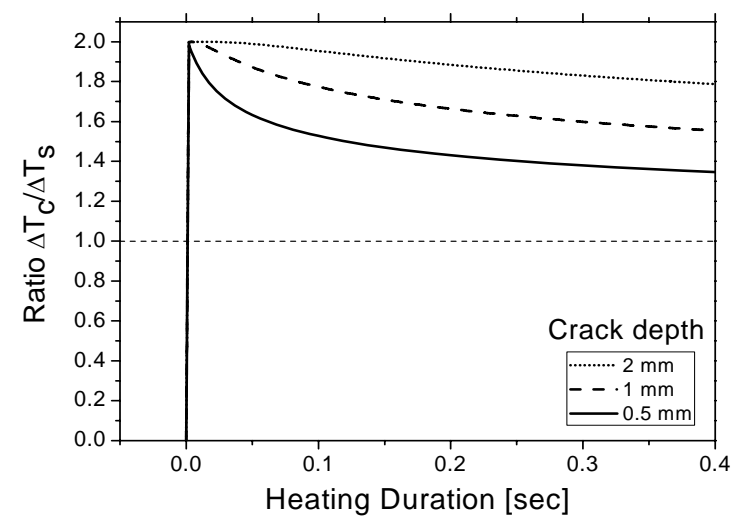

Figure 13: Ratio between the temperature increase in the corner of the crack and at the side of the sample as a function of the heating period for different crack depth values. The curves are calculated with the analytical model of $[7,8]$. 


\section{Summary}

A semi-analytical model has been presented, for calculating the eddy current and temperature distribution around a surface crack for the thermo-inductive testing method. Experimental and simulation results have been shown, consistently demonstrating that surface cracks can be identified with the thermo-inductive technique. After a short heating period in magnetic materials cracks are made visible by higher temperatures and in non-magnetic materials by lower temperatures. The results of the semi-analytical model are in good agreement with the simulation and with the experimental results, but further work is in progress for modelling the eddy current distribution more accurately.

\section{REFERENCES}

[1] Nipponn Steel Corp., Method of detecting a surface flaw of metallic material, Patent No US4109508, 1978.

[2] Elkem, Detection of flaws in metal members, Patent No Re32166, 1979.

[3] Elkem, Method of flaw detection in billets, Patent No US4480928, 1984.

[4] G.Busse, Verfahren zur phasenempfindlichen Darstellung eines effektmodulierten Gegenstandes, Patent No DE4203272, 1992.

[5] T.Sakagami,S.Kubo, Development of a new crack identification method based on singular current field using differential thermography, Proceedings of SPIE 3700 369-376, 1999.

[6] J.Bamberg, G.Erbeck, G.Zenzinger, EddyTherm: Ein Verfahren zur bildgebenden Rißprüfung metallischer Bauteile ZfP-Zeitung 68, p.60 - 62, 1999.

[7] B. Oswald-Tranta, Thermoinductive Investigations of Magnetic Materials for Surface Cracks, QIRT Journal Vol.1., p.33-46. 2004

[8] B. Oswald-Tranta, G.Wally, Thermo-inductive investigations of steel wires for surface cracks, ThermoSense XXVII, Orlando, Proceedings of SPIE Vol. 5782, p.245-254

[9] K.Simonyi, Theoretische Elektrotechnik, Barth Verlagsgesellschaft mbH, 1993.

[10] http://www.ansys.com 